\title{
Magnetic exchange-coupling effects in asymmetric trilayer structures of $\mathrm{MBE}$-grown $\mathrm{Co} / \mathrm{Cr} / \mathrm{Fe}$
}

\author{
Katharina Theis-Bröhl, Rainer Scheidt, Thomas Zeidler, Frank Schreiber, and Hartmut Zabel \\ Ruhr-University Bochum, Institut für Experimentalphysik/Festkörperphysik, D-44780 Bochum, Germany \\ Thomas Mathieu, Christoph Mathieu, ${ }^{*}$ and Burkard Hillebrands* \\ Universität Karlsruhe, Physikalisches Institut, Engesser Strasse 7, D-76128 Karlsruhe, Germany \\ (Received 1 June 1995; revised manuscript received 11 December 1995)
}

\begin{abstract}
We present results of anisotropy and exchange-coupling studies of asymmetric $\mathrm{Co} / \mathrm{Cr} / \mathrm{Fe}$ trilayers and superlattices grown by molecular beam epitaxy on $\mathrm{Cr}(001) / \mathrm{Mg}(001)$ buffers and substrates. The magnetic properties have been investigated using both the longitudinal magneto-optical Kerr effect and ferromagnetic resonance. The hysteresis data obtained from the trilayer system were fit to a theoretical model which contains both bilinear and biquadratic coupling. The effective in-plane anisotropy was found to be of fourfold symmetry with the same easy-axis orientation for both the Fe and Co layers. An analysis of the easy-axis hysteresis loops indicates long-period oscillatory coupling and also suggests a short periodic coupling. We show that weakly antiferromagnetically coupled asymmetric films might serve as potential candidates for improved spin-valve systems.
\end{abstract}

\section{INTRODUCTION}

Oscillatory exchange coupling has been discovered for different magnetic materials and a wide variety of nonmagnetic (NM) spacer materials over the last several years (for references see Ref. 1). The periods of the oscillations can be understood in terms of the electronic structure of the nonmagnetic spacer layer material. ${ }^{2}$ However, some spacer materials like $\mathrm{Cr}$ or $\mathrm{Mn}$ are not completely ' "nonmagnetic.' ' In the case of $\mathrm{Cr}$ an antiferromagnetic (AF) structure of the moments is formed below the Néel temperature together with a spontaneous incommensurate collinear spin density wave. Therefore the exchange interaction between the $\mathrm{Cr}$ atomic planes should affect the coupling behavior of the adjacent magnetic layers as well. For the case of $\mathrm{Fe} / \mathrm{Cr}(001)$, tight binding calculations ${ }^{3}$ suggest $\mathrm{AF}$ interactions and, in the case of bcc $\mathrm{Co} / \mathrm{Cr}(001)$, predict stable states for either ferromagnetic (FM) or AF alignment. Other calculations by Stoeffler et l. $^{4}$ predict parallel interactions in the case of the bcc $\mathrm{Co} / \mathrm{Cr}(001)$ interface. But the interfacial magnetic coupling is predicted to be not very strong. That means that an antiparallel coupling is allowed in the case of a $\mathrm{Co} / \mathrm{Cr}(001)$ interface whereas for $\mathrm{Fe} / \mathrm{Cr}$ the parallel coupling does not exist.

Experimental investigations on high quality $\mathrm{Fe} / \mathrm{Cr}(001)$ trilayers with short two monolayer (ML) period oscillations find the strongest $\mathrm{AF}$ maxima for even $\mathrm{Cr}$ spacer atomic layer (AL) numbers. These are $8 \mathrm{ML}$ reported by Purcell et $a .^{5}$ and $\approx 4 \mathrm{ML}$ reported by Demokritov et al. ${ }^{6}$ However, if the magnetic layers are different on either side of the $\mathrm{Cr}$ spacer, then the AF maxima are expected at odd AL numbers.

Noncoupled multilayers consisting of two different magnetic materials with different coercitivities (which are separated by thick nonmagnetic spacers) have been studied for the purpose of using them as spin-valve systems. ${ }^{7,8}$ These multilayers are magnetically uncoupled. However, an AF alignment of the moments can be obtained because of the different coercitivities of the two magnetic materials. Therefore, in the magnetic field range between the two flip fields a giant magnetoresistance can be found for some material combinations. $^{7-10}$

\section{SAMPLE PREPARATION}

To study the coupling behavior of a system similar to $\mathrm{Fe} / \mathrm{Cr}(001)$ but with a different second FM/NM interface a $\mathrm{Co} / \mathrm{Cr} / \mathrm{Fe}$ trilayer was grown with a wedge-shaped $\mathrm{Cr}$ interlayer. To grow the films a conventional 3-in. RIBER EVA 32 metal molecular beam epitaxy (MBE) system (equipped with two electron beam hearths and three ports for effusion cells) was used. One of the electron beam evaporators contains four rotable crucibles so that a total of eight different

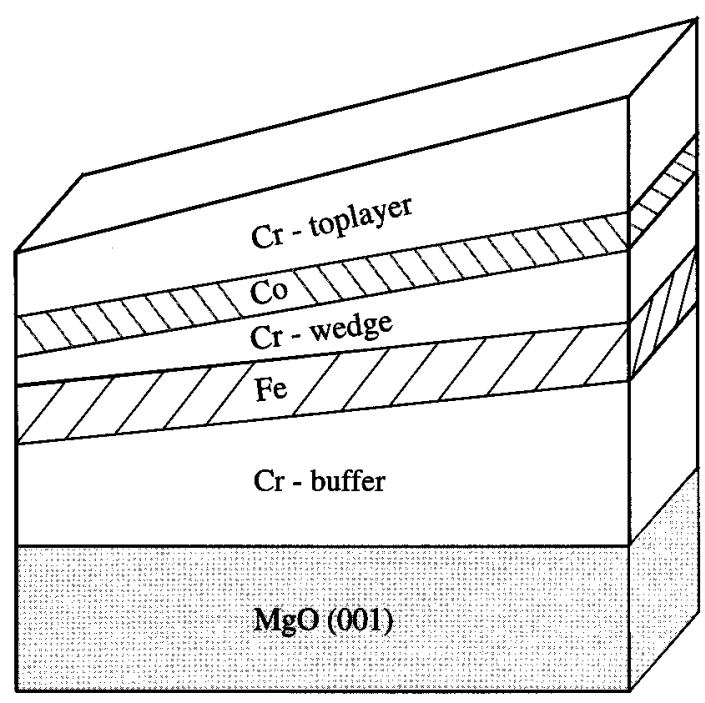

FIG. 1. Sequence of the $(\mathrm{Co} / \mathrm{Cr}$-wedge/Fe) film grown on $\mathrm{Cr} /$ $\mathrm{MgO}(001)$. 


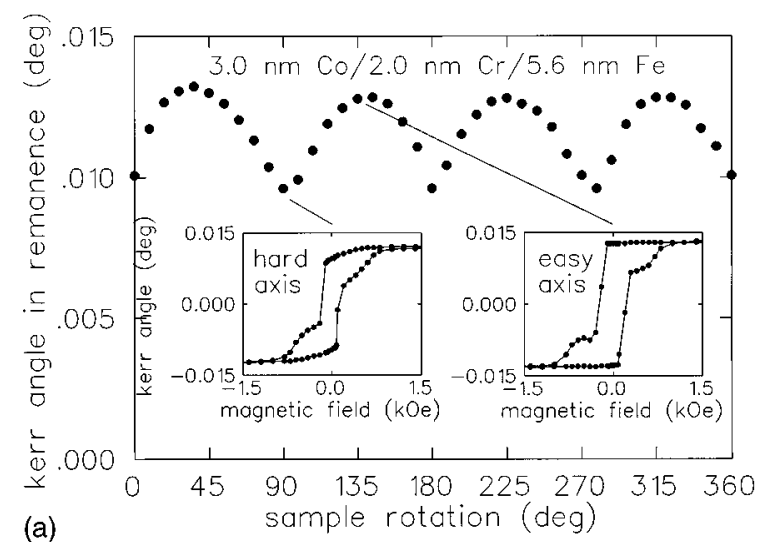

(a)

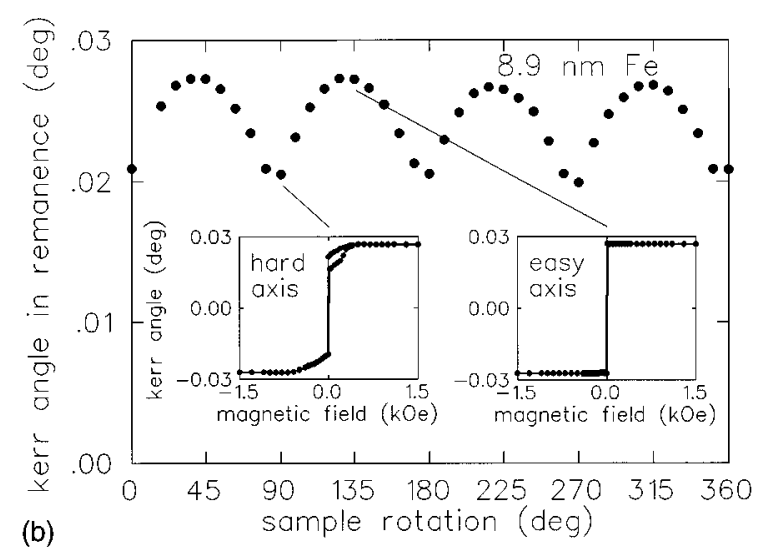

(b)

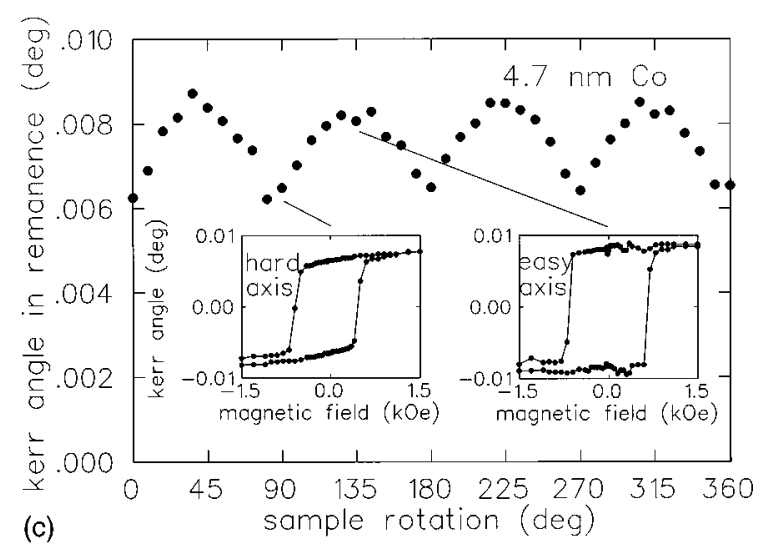

FIG. 2. Scans of the Kerr angle in remanence as a function of the sample rotation. Data from the MOKE hysteresis loops were taken during a complete sample rotation. The insets show the hardaxis (at minimum remanence) and easy-axis (at maximum remanence) hysteresis loops of the $(\mathrm{Co} / \mathrm{Cr}$-wedge/Fe) trilayer for $2.0 \mathrm{~nm}$ Cr layer thickness (a), a single 8.9-nm-thick Fe layer (b), and a 4.7-nm-thick Co-layer (c). Both single layers are embedded in $\mathrm{Cr}$.

materials can be evaporated from this system. The vacuum base pressure of the system is below $5 \times 10^{-9} \mathrm{~Pa}$ and the working pressure is better than $2 \times 10^{-8} \mathrm{~Pa}$.

The effusion cells contain $39-\mathrm{cm}^{3}$ crucibles providing a flux of a high stability. Therefore the film thickness could be established accurately by the evaporation time at a known growth rate. The flux of the materials which are evaporated from electron beam hearths is measured optically and controlled electronically during evaporation, and the shutters are

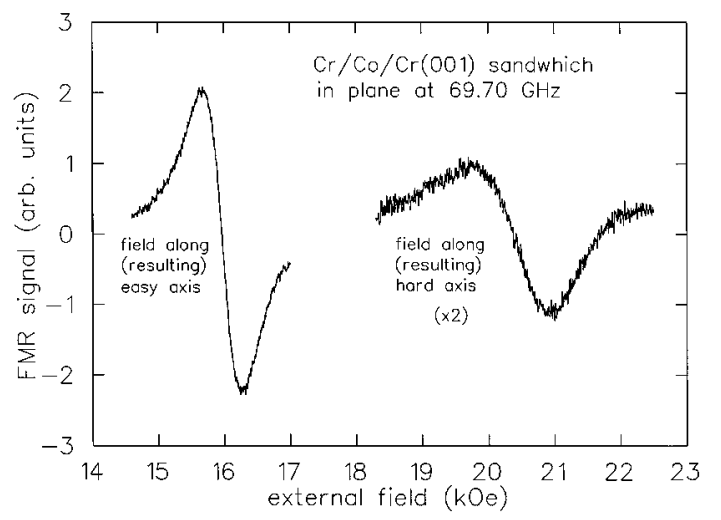

FIG. 3. FMR spectra of a 6.6-nm-thick single Co(11히) layer. The resonance line with the external field along the hard axis corresponds to the $c$ axis of one domain and perpendicular to the $c$ axis of the other domain (right); the resonance line with the external field along the easy axis is measured at $45^{\circ}$ with respect to the $c$ axes.

computer controlled. We evaporated Co from a $40-\mathrm{cm}^{3}$ electron beam hearth and $\mathrm{Fe}$ from one of the $14-\mathrm{cm}^{3}$ crucibles in the four-crucible gun. We used constant evaporation rates of $0.6 \mathrm{~nm} / \mathrm{min}$ for $\mathrm{Co}$ and $\mathrm{Fe}$ and different rates between 0.26 $\mathrm{nm} / \mathrm{min}$ and $1.6 \mathrm{~nm} / \mathrm{min}$ for the $\mathrm{Cr}$ spacer layer depending on the sample location. $\mathrm{Cr}$ was evaporated from an effusion cell with a pyrolytic graphite crucible. For the control of the $\mathrm{Cr}$ evaporation, only the effusion cell main shutter (which is in some distance from the cells) was used in order to ensure a stable temperature in the effusion cell during growth. The placement of the effusion cells is inclined at a certain angle to the 3-in. sample holder so that sample rotating is necessary to ensure a homogeneously thick film. Without rotation a linear gradient of the film thickness would be obtained over the sample holder. We used this linear gradient for designing our wedge-shaped $\mathrm{Cr}$ spacer.

To grow this system epitaxially we employed $\mathrm{MgO}(001)$ substrates. Since these substrates often have a surface that is insufficiently smooth for epitaxial growth, we subsequently repeated the commercial mechanical polishing of these substrates. With the help of ex situ small-angle x-ray scattering measurements and in situ reflection high-energy electron diffraction (RHEED) we were able to verify the surface quality of our $\mathrm{MgO}$ substrates before starting the growth. After introducing a freshly polished $\mathrm{MgO}$ sample into the vacuum chamber we outgassed and finally annealed it for more than $1 \mathrm{~h}$ at $1000{ }^{\circ} \mathrm{C}$.

Figure 1 shows the sequence of the sample we used for analyzing the anisotropy and coupling behavior. The first layer grown on the $\mathrm{MgO}$ substrate is a bcc $\mathrm{Cr}(001)$ buffer. The optimized substrate temperature is $500{ }^{\circ} \mathrm{C}$. Subsequently we annealed the buffer layer for $30 \mathrm{~min}$ at $750{ }^{\circ} \mathrm{C}$. The following layers are grown at a substrate temperature of 300 ${ }^{\circ} \mathrm{C}$. These consist of a 5.6-nm-thick bcc Fe(001) layer followed by the wedge-shaped $\mathrm{Cr}(001)$ spacer layer. The thickness of the spacer ranges from $0.5 \mathrm{~nm}$ and $3.0 \mathrm{~nm}$ over a sample length of $50 \mathrm{~mm}$. Fe and $\mathrm{Cr}$ are both bcc materials with similar lattice constants. Their lattices nearly perfectly match with a misfit of less than $1 \%$.

For the Co layer we have chosen a film thickness of only 

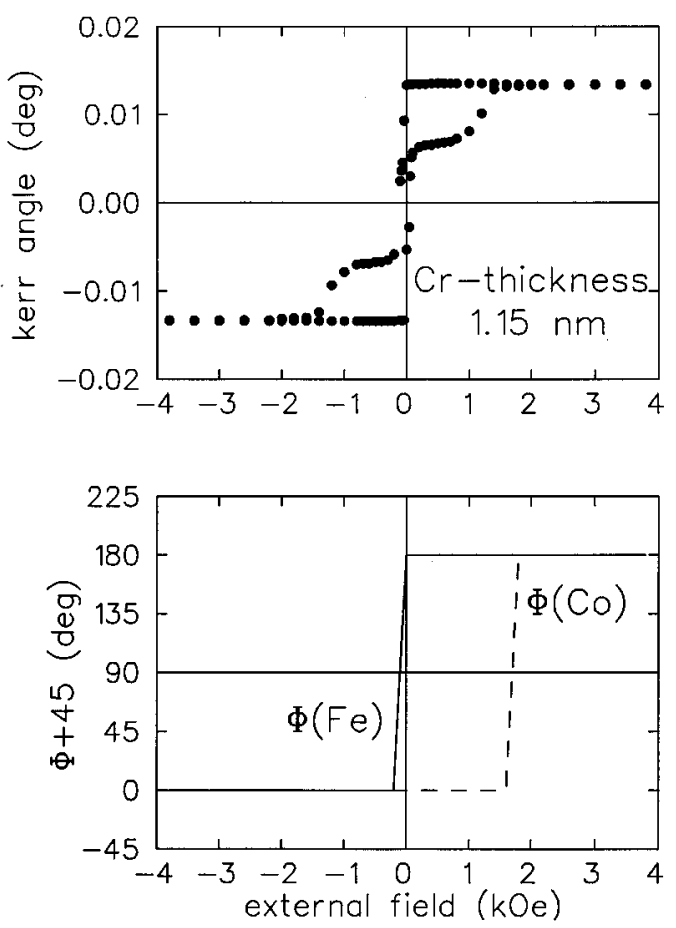

FIG. 4. The MOKE hysteresis loop for a Cr thickness of 1.15 $\mathrm{nm}$ measured at $45^{\circ}$ (easy-axis configuration), showing a weak $\mathrm{AF}$ behavior (above), and the field dependence of the angles $\Phi_{\mathrm{Fe}}$ and $\Phi_{\text {Co }}$ obtained from the fit (not shown) plotted for increasing field strength (below).

$3.0 \mathrm{~nm}$ because of the strong in-plane anisotropy. The Co layer grows in the hcp phase in the $(11 \overline{2} 0)$ orientation with the $c$ axis lying in the film plane. Since the uniaxial

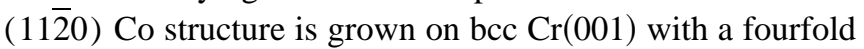
crystallographic symmetry, there are two equivalent orientations for the Co to grow with the $c$ axis parallel to the [110] and [1 $1 \overline{1} 0]$ axes of the $\mathrm{Cr}$, respectively. This results in a twined crystallographic domain structure ${ }^{11}$ On the Co film we have grown a $\mathrm{Cr}$ top layer to prevent oxidation of the Co film. Oxidation investigations of thin $\mathrm{Cr}$ layers have shown that $\mathrm{Cr}$ oxidizes not more than $1.0 \mathrm{~nm}$ deep at room temperature. $^{12}$

To check the total film thicknesses we used the thickness fringes of small-angle $\mathrm{x}$-ray scans at different sample positions. Although we have grown a wedge-shaped sample, we calculated the total film thicknesses from the peak positions of the maxima of the thickness fringes (we used only maxima at $2 \theta>2^{\circ}$ to minimize the size of the radiated area over the wedge). For these peak positions $\left(2 \theta>2^{\circ}\right)$ each calculated total film thickness deviates only slightly from the average thickness value. Unfortunately it was not possible to fit the small-angle x-ray data because of the strong angular depedence of the size of the radiated sample area for small angles $\left(2 \theta<2^{\circ}\right)$. By using additionally chemical x-ray fluorescence analysis the individual film thicknesses can be calculated for the different sample positions. This was possible because we have grown all individual Cr layers, including the buffer and the top layer, via the wedge shape. In this way the $\mathrm{Cr}$ growth rate exhibits a linear gradient across the sample which is constant during the entire film growth for
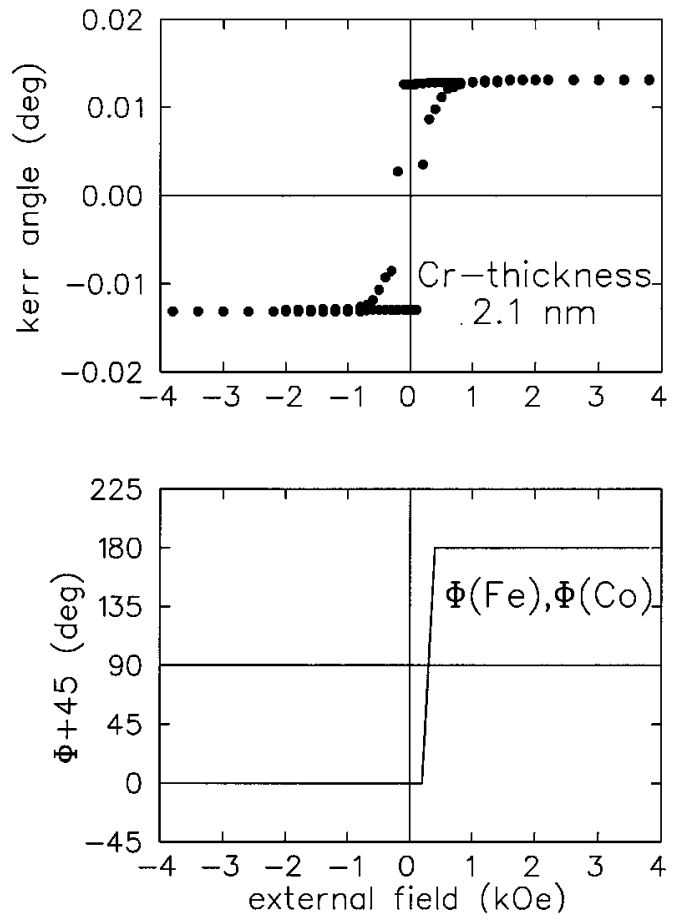

FIG. 5. The MOKE hysteresis loop for a Cr thickness of 2.10 $\mathrm{nm}$ measured at $45^{\circ}$ (easy-axis configuration), showing a weak FM behavior (above), and the field dependence of the angles $\Phi_{\mathrm{Fe}}$ and $\Phi_{C o}$ obtained from the fit (not shown) plotted for increasing field strength (below).

each specific point of the sample. Because of the large sample length $(50 \mathrm{~mm})$, the gradient over the already wedgeshaped buffer layer (ranging from 5 to $30 \mathrm{~nm}$ ) is not too large (1 ML increase in thickness at each $300 \mu \mathrm{m}$ lateral distance). With these Cr-growth rates we were able to estimate the $\mathrm{Cr}$ spacer thicknesses from the total Cr thicknesses taking into consideration the proportions of the $\mathrm{Cr}$-growth times.

With chemical $\mathrm{x}$-ray fluorescence analysis we also checked the proportions of the individual materials over the entire sample and found that the $\mathrm{Cr}$ spacer thickness shows the expected linear gradient.

The structural measurements did not include studies of the interface roughness or possible interdiffusion. However, fits to high-angle $\mathrm{X}$-ray diffraction data of $\mathrm{Co}(11 \overline{2} 0) / \mathrm{Cr}(001) \mathrm{su}-$ perlattices grown in the same MBE under the same conditions as the $\mathrm{Co} / \mathrm{Cr} / \mathrm{Fe}$ films should give reliable values for the interface roughness which was determined to $\sigma \approx 5 \AA .{ }^{13}$ Studies of $\mathrm{Fe} / \mathrm{Cr}$ superlattices (grown in a different chamber) give an even smaller value for the interface roughness of $\sigma \approx 3.5 \AA^{14}$

We have also grown samples with individual $\mathrm{Co}$ or $\mathrm{Fe}$ layers, respectively, embedded in $\mathrm{Cr}$ layers and superlattices with ten periodicities of the sequence of $(\mathrm{Cr} / \mathrm{Co} / \mathrm{Cr} / \mathrm{Fe})$. In these cases we used the sample rotation option during growth to get homogeneous $\mathrm{Cr}$ thicknesses over the films.

\section{MOKE MEASUREMENTS}

To study the magnetic properties of the $\mathrm{Co} / \mathrm{Cr} / \mathrm{Fe}$ sandwich sample we measured hysteresis loops with the 
magneto-optical Kerr effect (MOKE) in the longitudinal configuration as a function of the $\mathrm{Cr}$ interlayer thickness by moving the laser spot along the wedge-shaped sandwich. The MOKE setup is described elsewhere. ${ }^{15}$ The laser spot of a linear polarized He-Ne laser was focused to a diameter of $100 \mu \mathrm{m}$. We have chosen an incidence angle of the laser light of about $45^{\circ}$ to the sample surface. Therefore the measurements are sensitive to the in-plane component of the magnetization. The reflected laser beam passes through a modulator and a rotator using the magneto-optical Faraday effect to measure quantitatively the Kerr rotation angle. The relatively high modulation amplitude and the use of lock-in techniques provides an angular resolution of better than $10^{-4} \mathrm{deg}$.

For each $\mathrm{Cr}$ thickness we performed a complete in-plane sample rotation and we measured hysteresis loops at every $9^{\circ}$ of sample rotation to determine the easy and hard axes. For measuring the hysteresis loops we used a maximum magnetic field of $5.0 \mathrm{kOe}$. By plotting the Kerr angle in remanence as a function of sample orientation, indicated by the angle $\Phi_{\mathrm{H}}$ of the external field with respect to the $\mathrm{Cr}[001]$ in-plane direction, a fourfold anisotropy was obtained. In Fig. 2(a) we present such a plot for the $(\mathrm{Co} / \mathrm{Cr} / \mathrm{Fe})$ trilayer with $\mathrm{Cr}$ spacer thickness of $2.0 \mathrm{~nm}$. The hysteresis loops show a nearly uncoupled behavior [insets of Fig. 2(a)] for this case. We also show plots of an individual 8.9-nm-thick Fe layer [Fig. 2(b)] and an individual 4.7-nm-thick Co layer [Fig. 2(c)]. Note the much higher coercitivity of the Co layer.

The maxima of the Kerr angles in remanence mark the in-plane easy axes. For Fe films they correspond to the crystallographic [100] and [010] directions; this reflects the wellknown result of a positive cubic anisotropy parameter $K_{1}^{\text {cub }}$ (Ref. 16) in [001] oriented films. In the case of Co, a fourfold anisotropy was measured as well. The hard axes are found to be along the [110] and [1힐 axes which are parallel to the Co $c$ axes and thus parallel to the hard in-plane axes of the $\mathrm{Fe}$ film. This behavior was found recently for $\operatorname{Co}(11 \overline{2} 0)$ on $\mathrm{Cr}(001) / \mathrm{Nb}(001) / \mathrm{Al}_{2} \mathrm{O}_{3}(1 \overline{1} 02)$ by ferromagnetic resonance (FMR) measurements (see below and in Ref. 17).

In order to analyze the coupling behavior we performed MOKE hysteresis measurements at different points over the wedge-shaped trilayer sample. We discuss their behavior below.

\section{FMR MEASUREMENTS}

To investigate further the nature of the fourfold anisotropy of $\mathrm{Co}$ in these samples FMR measurements were carried out on $(\mathrm{Cr} / \mathrm{Co} / \mathrm{Cr} / \mathrm{Fe})$ superlattices as well as on individual $\mathrm{Fe}$ and $\mathrm{Co}$ layers embedded in (001)Cr layers using the same setup as described in Ref. 17. All samples were grown on a $\mathrm{Cr}(001)$ buffer layer on $\mathrm{MgO}(001)$ substrates.

All samples show an almost perfect fourfold in-plane anisotropy. This result can be readily understood if one assumes equal film areas for both in-plane growth orientations of Co. The uniaxial anisotropy contribution of hcp Co with the $c$ axis as the easy axis is averaged out due to the $90^{\circ}$ orientation of the $c$ axes of the two growth orientations. The next higher anisotropy contribution in hep symmetry, $K_{2}^{\mathrm{hcp}}$, which is of fourfold symmetry, is observed as the lowest-order contribution. ${ }^{17}$
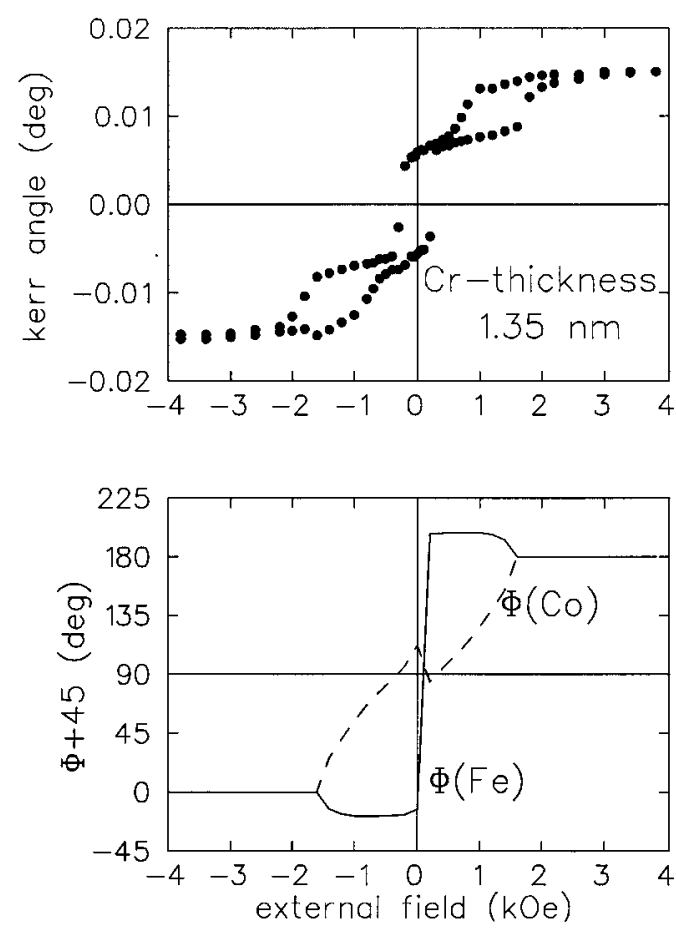

FIG. 6. The MOKE hysteresis loop for a Cr thickness of 1.35 $\mathrm{nm}$ measured at $45^{\circ}$ (easy-axis configuration), showing a strong AF behavior (above), and the field dependence of the angles $\Phi_{\mathrm{Fe}}$ and $\Phi_{\text {Co }}$ obtained from the fit (not shown) plotted for increasing field strength (below).

To discuss the Co behavior in more detail we show the resonance spectra of a single $\operatorname{Co}(11 \overline{2} 0)$ layer with a thickness of $6.6 \mathrm{~nm}$ (see Fig. 3). A purely fourfold anisotropy can be concluded from the observation of about the same spectra after each $90^{\circ}$ sample rotation. To estimate the areal difference of the portion of both growth orientations we use the difference of the line positions of FMR spectra at $\Phi_{H}$ and $\left(\Phi_{H}+90^{\circ}\right)$ which is less than $0.1 \mathrm{kOe}$. From this value the difference is estimated to be less than $\approx 2 \%$ using the relations given in Ref. 17.

Only one resonance line at each easy-axis orientation was found in the FMR spectra of the Co layers. This indicates that the crystallographic Co growth domains are much smaller in size than the magnetic domains; i.e., the crystallographic domains can be treated in the limit of strong magnetic coupling. ${ }^{17}$ In the opposite case, two lines would be observed. For the case found here, and with a nearly 1:1 proportion of the Co-growth domains, the resulting anisotropy energy is $F_{\text {ani }}^{\text {eff }}=\frac{1}{2} F_{\text {ani }}^{\text {eff }}(\Phi)+\frac{1}{2} F_{\text {ani }}^{\text {eff }}\left(\Phi+90^{\circ}\right)$ with contributions from the two growth domains of $F_{\text {ani }}^{\mathrm{eff}}(\Phi)$ and $F_{\text {ani }}^{\text {eff }}\left(\Phi+90^{\circ}\right)$. Here $\Phi$ is the in-plane angle of the direction of magnetization measured against the in-plane $\mathrm{Cr}[001]$ axis. The resulting in-plane anisotropy is fourfold and has the same shape as that for cubic in-plane anisotropies. The hcp anisotropy constant $K_{2}$ is in this case the relevant parameter for the anisotropy expression. If $K_{2}>0$, the easy axis will then be found between the two $c$ axes. Therefore, neglecting the out-of-plane $\Theta$ dependence, we can write the anisotropy energy (as for the Co case ${ }^{17}$ ) 
TABLE I. Results of the FMR measurements for individual Co and Fe layers and superlattices. For the purpose of comparison, also the Fe bulk values are given.

\begin{tabular}{lcccccc}
\hline \hline Sample & $t_{\mathrm{Fe}}$ & $t_{\mathrm{Co}}$ & $4 \pi M_{\text {eff }}$ & $4 \pi M_{\text {eff }}^{*}$ & $2 K_{1} / M$ & $K_{2}^{\text {eff }} / M$ \\
\hline Co sandwich & & $6.6 \mathrm{~nm}$ & & $14.5 \mathrm{kOe}$ & & $1.5 \mathrm{kOe}$ \\
Co sandwich & & $4.7 \mathrm{~nm}$ & & $14.5 \mathrm{kOe}$ & & $1.8 \mathrm{kOe}$ \\
$(\mathrm{Cr} / \mathrm{Co} / \mathrm{Cr} / \mathrm{Fe})_{10}$ & $3.2 \mathrm{~nm}$ & $2.6 \mathrm{~nm}$ & $21.0 \mathrm{kOe}$ & & $0.40 \mathrm{kOe}$ & \\
$(\mathrm{Cr} / \mathrm{Co} / \mathrm{Cr} / \mathrm{Fe})_{10}$ & $2.4 \mathrm{~nm}$ & $2.0 \mathrm{~nm}$ & $21.6 \mathrm{kOe}$ & & $0.53 \mathrm{kOe}$ & \\
$\mathrm{Fe}$ sandwich & $3.0 \mathrm{~nm}$ & & $21.84 \mathrm{kOe}$ & & $0.54 \mathrm{kOe}$ & \\
Fe sandwich & $8.9 \mathrm{~nm}$ & & $21.98 \mathrm{kOe}$ & & $0.60 \mathrm{kOe}$ & \\
Fe bulk & & $21.49 \mathrm{kOe}$ & & $0.55 \mathrm{kOe}$ & \\
\hline \hline
\end{tabular}

$$
\begin{aligned}
F_{\text {ani }}^{\mathrm{eff}} & =\frac{1}{8} K_{2} \cos \left[4\left(\Phi+45^{\circ}\right)\right] \\
& =-K_{2} \sin ^{2}\left(\Phi+45^{\circ}\right) \cos ^{2}\left(\Phi+45^{\circ}\right)+\text { const } \\
& =K_{2} \sin ^{2}(\Phi) \cos ^{2}(\Phi)+\text { const. }
\end{aligned}
$$

The angular offset of $45^{\circ}$ accounts for the rotation of the $c$ axes from the [100] axes. Table I shows the results of the anisotropy parameters including the effective magnetization which is defined as

$$
4 \pi M_{\mathrm{eff}}^{*}=4 \pi M_{s}-2 \frac{2 K_{s}}{2 M t_{\mathrm{Co}}}+\frac{K_{1}^{\mathrm{hcp}}}{M}+\frac{2 K_{2}^{\mathrm{hcp}}}{M}
$$

for the (11 $\overline{2} 0)$-oriented Co layers (following the notation of Ref. 17). For the present thickness range, the $K_{s}$ term is of minor importance. The results for the $\mathrm{Fe}(001)$ individual layers show almost bulklike behavior. $4 \pi M_{\text {eff }}$ for Fe is defined in the usual manner collecting all $\sin ^{2} \Theta$ contributions of the energy expression. The behavior of the $(\mathrm{Cr} / \mathrm{Co} / \mathrm{Cr} / \mathrm{Fe})$ superlattices is apparently dominated by the Fe layers. The result of the fits of the FMR data for both Co samples yields $4 \pi M_{\text {eff }}=14.5 \mathrm{kG}$. This value is in good agreement with the bulk value, taking into consideration the bulk value of $K_{1} / M \approx 3 \mathrm{kOe}$ and $2 K_{2} / M \approx 1-2 \mathrm{kOe}$ and neglecting surface anisotropy effects for this thickness.

\section{DISCUSSION}

To analyze the MOKE hysteresis loops of the wedgedshaped $\mathrm{Co} / \mathrm{Cr} / \mathrm{Fe}$ sandwich (see Figs. 4-7) we tried to fit our data of the hard- and easy-axis loops. We assumed the following expression for the free energy, neglecting constant terms:

$$
\begin{aligned}
F_{\mathrm{mag}}= & {\left[-\mu_{0} M^{\mathrm{Fe}} H \cos \left(\Phi_{\mathrm{Fe}}-\Phi_{H}\right)+K_{1}^{\mathrm{Fe}} \sin ^{2} \Phi_{\mathrm{Fe}} \cos ^{2} \Phi_{\mathrm{Fe}}\right] t_{\mathrm{Fe}} } \\
& +\left[-\mu_{0} M^{\mathrm{Co}} H \cos \left(\Phi_{\mathrm{Co}}-\Phi_{H}\right)\right. \\
& \left.+K_{2}^{\mathrm{Co}} \sin ^{2} \Phi_{\mathrm{Co}} \cos ^{2} \Phi_{\mathrm{Co}}\right] t_{\mathrm{Co}} \\
& -2 A_{12} \cos \left(\Phi_{\mathrm{Fe}}-\Phi_{\mathrm{Co}}\right)-2 B_{12} \cos ^{2}\left(\Phi_{\mathrm{Fe}}-\Phi_{\mathrm{Co}}\right) .
\end{aligned}
$$

To minimize the free energy a simplex algorithm was used. The algorithm guarantees that after every field step the next local minimum is searched.

For very small Cr layer thicknesses $\left(t_{\mathrm{Cr}}=0.55-0.75 \mathrm{~nm}\right)$ a simultaneous fit to the data of the easy and hard axes was possible. Unfortunately, the fits to the data for thicker $\mathrm{Cr}$ spacers do not yield a uniform set of parameters for both axes. The theoretically expected steps in the hysteresis loops are too much rounded out in our experimental data. Responsible for this are probably domain processes which are not included in our model. For small $\mathrm{Cr}$ thicknesses the strong ferromagnetic coupling suppresses the formation of domains.

For Cr spacers thicker than $0.75 \mathrm{~nm}$ fits to the data of the MOKE hysteresis loops with the external field applied along the easy-axis direction at $135^{\circ}$ provide information on the coupling constants as well as on the alignment of the Fe and Co moments. These fits to the easy-axis loops are in reasonable agreement mainly for those parts of the loops at which no domain nucleation processes and domain wall movements determine the magnetization behavior.

Taking into consideration the different thicknesses (5.6 $\mathrm{nm}$ for $\mathrm{Fe}$ and $3.0 \mathrm{~nm}$ for $\mathrm{Co}$ ), the product of the saturation magnetization (assuming bulklike behavior) and the layer thickness $\left(M_{s} t\right)$ in the external field term of the free energy is about twice as high for the Fe layer as for the Co layer. Therefore the behavior of the Fe layer is expected to be influenced much stronger by the external field than that of the Co layer. This is demonstrated by the results of the fits for $\Phi_{\mathrm{Fe}}$ and $\Phi_{\mathrm{Co}}$ of the easy-axis hysteresis loops (see Figs. 4-7).

In particular, the Fe moments flip already at small (reverse) magnetic fields while the moments in the Co layer remain in the original direction. Apparently, the magnetization of the Co layer follows the rotation of the Fe layer and not the external field. In addition the much higher coercitivity of individual Co layers (see Fig. 2) leads to the conclusion that Co must undergo a much more complicated domain structure. Due to the different magnetic behavior of $\mathrm{Fe}$ and Co [see Figs. 2(b) and (c)], a nearly uncoupled $\mathrm{Co} / \mathrm{Cr} / \mathrm{Fe}$ trilayer shows hysteresis loops which are close to those shown in Figs. 2(a) for $t_{\mathrm{Cr}}=2.0 \mathrm{~nm}$.

The fits for the $\mathrm{Cr}$ thickness of $t_{\mathrm{Cr}}=2.0 \mathrm{~nm}$ reveal the absence of bilinear coupling $\left(A_{12}=0\right)$ and a weak biquadratic coupling $\left(B_{12}=-0.01 \mathrm{~mJ} / \mathrm{m}^{2}\right)$. Because the high coercitivity of the Co layer is not included in our model, this biquadratic coupling value of $B_{12}=-0.01 \mathrm{~mJ} / \mathrm{m}^{2}$ might represent the Co coercitivity and not a "real" biquadratic coupling.

The shapes of the easy-axis hysteresis loops for a weak AF coupling differ from those with a nearly uncoupled behavior mainly by a longer step in the loop for the former case. In Fig. 4 we present an easy-axis hysteresis loop for weakly AF coupled $\mathrm{Co}$ and $\mathrm{Fe}$ layers at a $\mathrm{Cr}$ thickness 

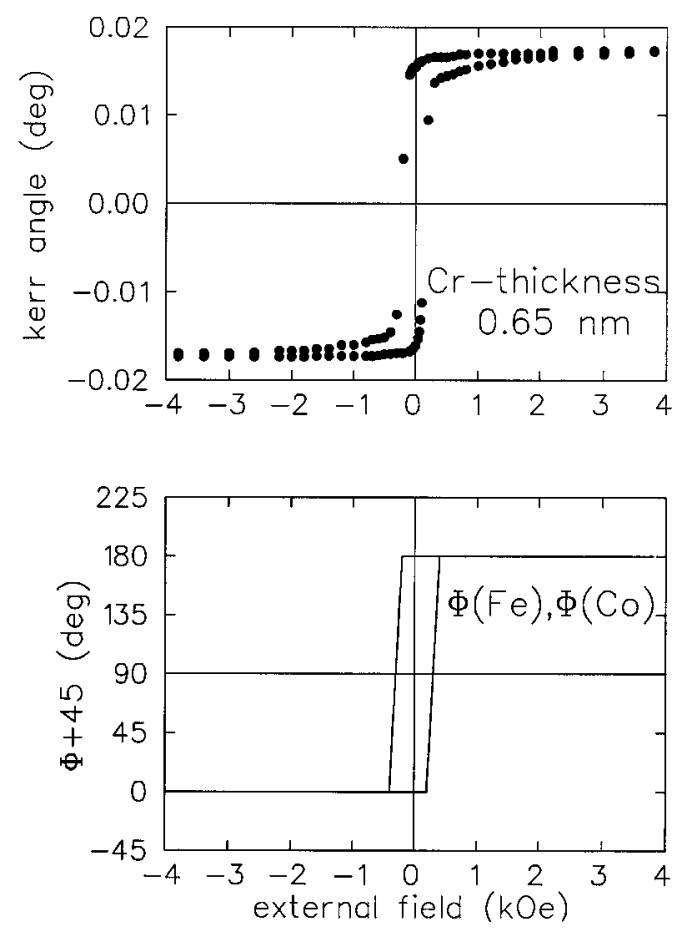

FIG. 7. The MOKE hysteresis loop for a Cr thickness of 0.65 $\mathrm{nm}$ measured at $45^{\circ}$ (easy-axis configuration), showing a FM behavior (above), and the field dependence of the angles $\Phi_{\mathrm{Fe}}$ and $\Phi_{\text {Co }}$ obtained from the fit (not shown) plotted for increasing field strength (below).

$t_{\mathrm{Cr}}=1.15 \mathrm{~nm}$ (with the fit results $A_{12}=-0.03 \mathrm{~mJ} / \mathrm{m}^{2}$, $B_{12}=-0.01 \mathrm{~mJ} / \mathrm{m}^{2}$ ). In comparison, for a weak FM coupling strength the length of the step decreases or the step nearly vanishes (see Fig. 5). It is interesting to note that the length of the step is very sensitive to the coupling behavior. Slight variations of the coupling constants in the case of a weak coupling behavior (FM or AF) produce drastic changes of the step length [compare Figs. 4 and 5 and insets of Figs. 2(a) and 8]. Also the flip field of the Fe moments undergoes changes with the coupling characteristics. A systematic change can be obtained in the case of a weak coupling beavior when the flip of the $\mathrm{Fe}$ moments occurs before the Co

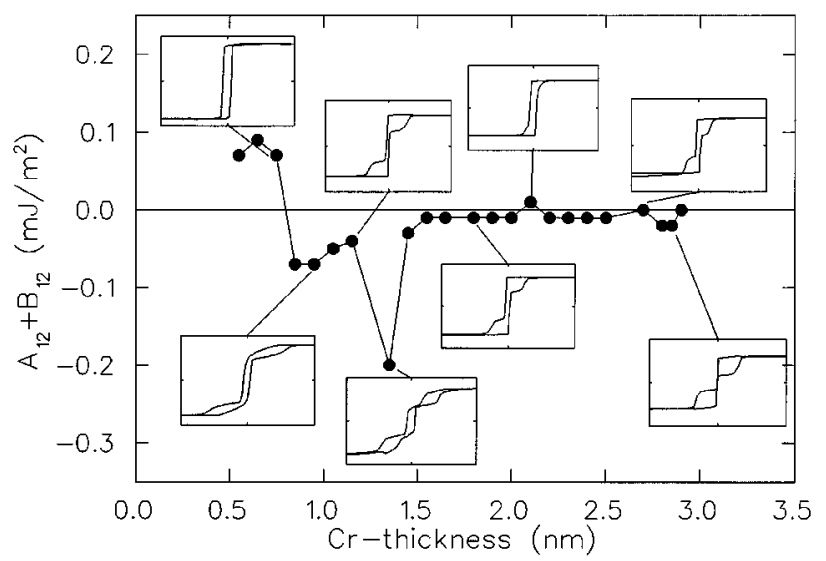

FIG. 8. Results of our fits to the data for $A_{12}+B_{12}$ as a function of the $\mathrm{Cr}$ spacer thickness. The insets show the shapes of the hysteresis loops measured at different $\mathrm{Cr}$ thicknesses. moments change their alignment. For a weak AF coupling the flip of the Fe moments may occur at slightly negative magnetic fields, at zero field, or very small positive fields. For zero coupling or a weak FM coupling the flip of the Fe moments occurs at slightly higher positive magnetic fields.

The behavior of these weak AF coupled asymmetric trilayers is very interesting for use as spin valves. By choosing the proper $\mathrm{Cr}$ spacer thickness the flip of the Fe moments can be tuned to occur at zero or at very small magnetic fields. The clear step in the hysteresis loops after the flip of the $\mathrm{Fe}$ moments occurs indicates the AF alignment of the moments of the $\mathrm{Fe}$ and Co layers over a wide magnetic field range ( $\delta \mathrm{H} \approx 1 \mathrm{kOe}$ for the hysteresis loop shown in Fig. 4). The weak AF coupling should also be interesting for other spinvalve systems since it could solve the problem of finding a material with an extremely small coercitivity for spin-valve systems. By tuning the first spin flip to about zero field by using the proper spacer thickness the expected flip behavior for ideal spin valves could be reached.

In all cases of our $(\mathrm{Co} / \mathrm{Cr} / \mathrm{Fe})$ trilayer with weak coupling characteristics, the Co moments first remain in their original alignment after the flip of the Fe moments occurs. With increasing field domain and/or rotation processes of the moments occur. For a larger AF coupling the Co moments change their alignment before the flip of the $\mathrm{Fe}$ moments occurs (see Fig. 6). For a Cr thickness of $1.35 \mathrm{~nm}$ the strongest $\mathrm{AF}$ coupling constants were found $\left(A_{12}=-0.12\right.$ $\mathrm{mJ} / \mathrm{m}^{2}$ and $\left.B_{12}=-0.08 \mathrm{~mJ} / \mathrm{m}^{2}\right)$. Note that a high biquadratic coupling constant $B_{12}$ (compared to $A_{12}$ ) is obtained. For $\mathrm{Cr}$ thicknesses between $0.85 \mathrm{~nm}$ and $1.05 \mathrm{~nm}$, the shapes of the hysteresis loops again suggest a strong AF coupling. For this region we found coupling constants of $\left(A_{12}+\right.$ $B_{12}=-0.05$ to $-0.07 \mathrm{~mJ} / \mathrm{m}^{2}$ ). In this case the biquadratic coupling constant $B_{12}$ is much higher than the bilinear one $A_{12}$ (see Table II).

For a strong FM coupling we find hysteresis loops without any steps and a clear FM shape. In this case the Co layer couples so strongly to the Fe with FM moment alignment that the magnetization processes of both materials occur together. Such loops can be obtained at $t_{\mathrm{Cr}}=0.55 \mathrm{~nm}$ and $t_{\mathrm{Cr}}=0.75 \mathrm{~nm}$. At $t_{\mathrm{Cr}}=0.65 \mathrm{~nm}$ the shape of the hysteresis loops suggests that the Co moments do not reach complete saturation after the flip (see Fig. 7). For all three Cr layer thicknesses the fits give a positive coupling constant $A_{12}=0.07-0.09 \mathrm{~mJ} / \mathrm{m}^{2}$.

In Fig. 8 we present the results of our simulations for the coupling constants $A_{12}+B_{12}$ as a function of the Cr spacer thickness. A long-period coupling oscillation with a period of $10-11 \mathrm{ML}$ is deduced from the two AF maxima at $\approx 9$ $\mathrm{ML}$ and $\approx 20 \mathrm{ML}$ and from the FM maxima at 4-5 ML and $\approx 15 \mathrm{ML}$. The second-long period $\mathrm{AF}$ maxima at $t_{\mathrm{Cr}}=2.85$ $\mathrm{nm}\left(A_{12}=-0.01 \mathrm{~mJ} / \mathrm{m}^{2}\right.$ and $\left.B_{12}=-0.01 \mathrm{~mJ} / \mathrm{m}^{2}\right)$ also can be deduced clearly from the shapes of the hysteresis loops in this $\mathrm{Cr}$ region (see insets of Fig. 8). Also a 2-ML shortperiod coupling might exist in this system. We obtained two strong $\mathrm{AF}$ maxima at $\approx 7 \mathrm{ML}$ and $\approx 9 \mathrm{ML}$, separated by a region with weak coupling characteristics. But in other $\mathrm{Cr}$ thickness regions no short-period coupling can be found.

The odd $\mathrm{Cr}$ atomic layer number suggests a ferromagnetic $\mathrm{Co} / \mathrm{Cr}$ interface exchange coupling (parallel alignment of moments), assuming an antiferromagnetic exchange for the $\mathrm{Fe} / \mathrm{Cr}$ interface. 
TABLE II. Results of the fits to the MOKE hysteresis loops for Cr thicknesses.

\begin{tabular}{lcccc}
\hline \hline$t_{\mathrm{Cr}}(\mathrm{nm})$ & $t_{\mathrm{Cr}}(\mathrm{ML})$ & $A_{12}\left(\mathrm{~mJ} / \mathrm{m}^{2}\right)$ & $B_{12}\left(\mathrm{~mJ} / \mathrm{m}^{2}\right)$ & $A_{12}+B_{12}\left(\mathrm{~mJ} / \mathrm{m}^{2}\right)$ \\
\hline $0.55 \mathrm{~nm}$ & $3.8 \mathrm{ML}$ & +0.07 & 0 & +0.07 \\
$0.65 \mathrm{~nm}$ & $4.5 \mathrm{ML}$ & +0.09 & 0 & +0.09 \\
$0.75 \mathrm{~nm}$ & $5.2 \mathrm{ML}$ & +0.07 & 0 & +0.07 \\
$0.85 \mathrm{~nm}$ & $5.9 \mathrm{ML}$ & 0 & -0.07 & -0.07 \\
$0.95 \mathrm{~nm}$ & $6.6 \mathrm{ML}$ & -0.02 & -0.05 & -0.07 \\
$1.05 \mathrm{~nm}$ & $7.3 \mathrm{ML}$ & 0 & -0.05 & -0.05 \\
$1.15 \mathrm{~nm}$ & $8.0 \mathrm{ML}$ & -0.03 & -0.01 & -0.04 \\
$1.35 \mathrm{~nm}$ & $9.4 \mathrm{ML}$ & -0.12 & -0.08 & -0.20 \\
$1.45 \mathrm{~nm}$ & $10.0 \mathrm{ML}$ & 0 & -0.03 & -0.03 \\
$1.55-2.00 \mathrm{~nm}$ & $10.8-13.9 \mathrm{ML}$ & 0 & -0.01 & -0.01 \\
$2.10 \mathrm{~nm}$ & $14.6 \mathrm{ML}$ & +0.02 & -0.01 & +0.01 \\
$2.20-2.50 \mathrm{~nm}$ & $15.3-17.4 \mathrm{ML}$ & 0 & -0.01 & -0.01 \\
$2.70 \mathrm{~nm}$ & $18.8 \mathrm{ML}$ & 0 & 0 & 0 \\
$2.80-2.85 \mathrm{~nm}$ & $19.4-19.8 \mathrm{ML}$ & -0.01 & -0.01 & -0.02 \\
$2.90 \mathrm{~nm}$ & $20.1 \mathrm{ML}$ & 0 & 0 & 0 \\
\hline \hline
\end{tabular}

In the case of $\mathrm{Fe} / \mathrm{Cr}$, biquadratic coupling can be observed mostly in a region between the AF and the FM maxima (see Ref. 18). But our fits to the data for the $\mathrm{Co} / \mathrm{Cr} / \mathrm{Fe}$ trilayer system suggest that the highest negative biquadratic coupling constant $B_{12}$ occurs at the strong AF maxima. As previously mentioned the coupling constant $B_{12}=-0.01 \mathrm{~mJ} / \mathrm{m}^{2}$ found (from the simulations) for most of the hysteresis loops in the case of a weak coupling may represent the coercitivity of the Co layer. The strong biquadratic coupling constants found at the AF maxima should be reduced by this value. This does not influence the main behavior.

\section{CONCLUSION}

In conclusion, we have studied the magnetic properties of $(\mathrm{Co} / \mathrm{Cr} / \mathrm{Fe})$ samples, as well as individual $\mathrm{Co}$ and Fe layers, by the MOKE and FMR. We suggest coupled crystallographic $(11 \overline{2} 0)$ Co domains with an almost 1:1 distribution of both domains causing a fourfold anisotropy behavior of the Co layer, with similar characteristics as for the Fe layer. The MOKE hysteresis loops show an indication of a long-period (10-11 ML) and a short-period (2 ML) exchange coupling oscillation, with the AF maxima at odd numbers of AL's of the $\mathrm{Cr}$ spacer. The strongest AF maximum (with a coupling constant of $-0.20 \mathrm{~mJ} / \mathrm{m}^{2}$ ) was found at $9 \mathrm{ML}$. The fits to the data also reveal a high biquadratic constant whenever the AF coupling shows maxima.

In the case of a zero or a weak coupling behavior both magnetic materials ( $\mathrm{Fe}$ and $\mathrm{Co}$ ) show different flip fields. After the first flip (Fe) an AF alignment between the Fe and the Co moments can be obtained. The flip of the Fe moments (the material with the smaller coercitivity) can be tuned to about zero field by choosing the proper $\mathrm{Cr}$ spacer layer thickness in the case of a weak AF coupling. Those asymmetric films with weak AF coupling behavior can be used as ideal spin-valve systems.

\section{ACKNOWLEDGMENTS}

The authors wish to thank W. Oswald, J. Podschwadek, and P. Stauche for technical help. We wish also to thank Z. Frait (Prague) for the use of his FMR spectrometer for additional measurements. The work was supported by the Deutsche Forschungsgemeinschaft through SFB 166. One of us (K.T.B.) thanks M. B. Salamon for helpful discussions and C. P. Flynn for hospitality and acknowledges partial support from the Physics Department at the University of Illinois at Urbana-Champaign.
*Present address: Fachbereich Physik, Universität Kaiserslautern, Erwin-Schrödinger-Straße 46, 67663 Kaiserslautern, Germany.

${ }^{1}$ M.D. Stiles, Phys. Rev. B 48, 7738 (1993).

${ }^{2}$ K.B. Hathaway, in Ultrathin Magnetic Structures II, edited by B. Heinrich and J.A.C. Bland (Springer-Verlag, Berlin, 1994).

${ }^{3}$ J.H. Hasegawa, Phys. Rev. B 42, 2368 (1990); 43, 10803 (1990).

${ }^{4}$ D. Stoeffler and G. Gautier, J. Magn. Magn. Mater. 104-107, 1819 (1992); Phys. Rev. B 44, 10389 (1991); Surf. Sci. 251/ 252, 31 (1991).

${ }^{5}$ S.T. Purcell, W. Folkerts, M.T. Johnson, N.W.E. McGee, K. Jager, J. Aan de Steege, W.P. Zeper, and P. Grünberg, Phys. Rev. Lett. 67, 903 (1991).

${ }^{6}$ S. Demokritov, J.A. Wolf, P. Grünberg, and W. Zinn, in Mag- netic Thin Films, Multilayers and Surfaces, edited by S.S.P. Parkin, MRS Symposia Proceedings No. 231 (Materials Research Society, Pittsburgh, 1991).

${ }^{7}$ T. Shinjo and H. Yamamoto, J. Phys. Soc. Jpn. 59, 3061 (1990); H. Yamamoto, T. Okuyama, H. Dohnomae, and T. Shinjo, J. Magn. Magn. Mater. 99, 243 (1991).

${ }^{8}$ B. Dieny, V.S. Speriosu, S.S.P. Parkin, B.A. Gurnay, D.R. Wilhoit, and D. Mauri, Phys. Rev. B 43, 1297 (1991).

${ }^{9}$ P.P. Freitas, J.L. Leal, T.S. Plaskett, and L.V. Melo, J. Appl. Phys. 75, 6480 (1994).

${ }^{10}$ H. Hosoya, M. Komoto, and Y. Sugita, J. Magn. Soc. Jpn. 18, 341 (1994).

${ }^{11}$ N. Metoki, W. Donner, and H. Zabel, Phys. Rev. B 49, 17351 
(1994); W. Donner, N. Metoki, A. Abromeit, and H. Zabel, Phys. Rev. B 48, 14745 (1993).

${ }^{12}$ A. Stierle (private communication).

${ }^{13}$ W. Donner, Ph.D. thesis, Ruhr-Universiät Bochum, Germany, 1994.

${ }^{14}$ A. Schreyer, J.F. Ankner, Th. Zeidler, H. Zabel, R. Schäfer, J.A. Wolf, P. Grünberg, and C.F. Majkrzak, Phys. Rev. B 52, 16066 (1995).

${ }^{15}$ N. Metoki, Th. Zeidler, A. Stierle, K. Bröhl, and H. Zabel, J.
Magn. Magn. Mater. 118, 57 (1993).

${ }^{16}$ Th. Mhüge, Th. Zeidler, Q. Wang, Ch. Morawe, N. Metoki, and H. Zabel, J. Appl. Phys. 77, 1055 (1995).

${ }^{17}$ F. Schreiber, Z. Frait, Th. Zeidler, N. Metoki, W. Donner, H. Zabel, and J. Pelzl, Phys. Rev. B 51, 2920 (1995).

${ }^{18}$ M. Rührig, R. Schäfer, A. Hubert, R. Mosler, J.A. Wolf, S. Demokritov, and P. Grünberg, Phys. Status Solidi A 125, 635 (1991). 